\title{
Hoher Blutdruck bessert die Prognose auf der kardiologischen Intensivstation
}

\author{
Hoher Blutdruck verschlechtert die \\ Prognose. Doch wer mit akuten \\ präkordialen Schmerzen auf die \\ Intensivstation kommt, hat bessere \\ Aussichten bei hohem systolischem \\ Blutdruck.
}

— Eine Datenbank in Schweden registriert die Aufnahmen aller Patienten auf alle medizinischen Intensivstationen des Landes. In den Jahren 1997 bis 2006 waren darunter 119151 mit akuten Herzschmerzen. Bei der Aufnahme lagen die Quartilen des systolischen Blutdrucks unter 128, bei 128-144 bzw. 145-162 und über $162 \mathrm{mmHg}$.

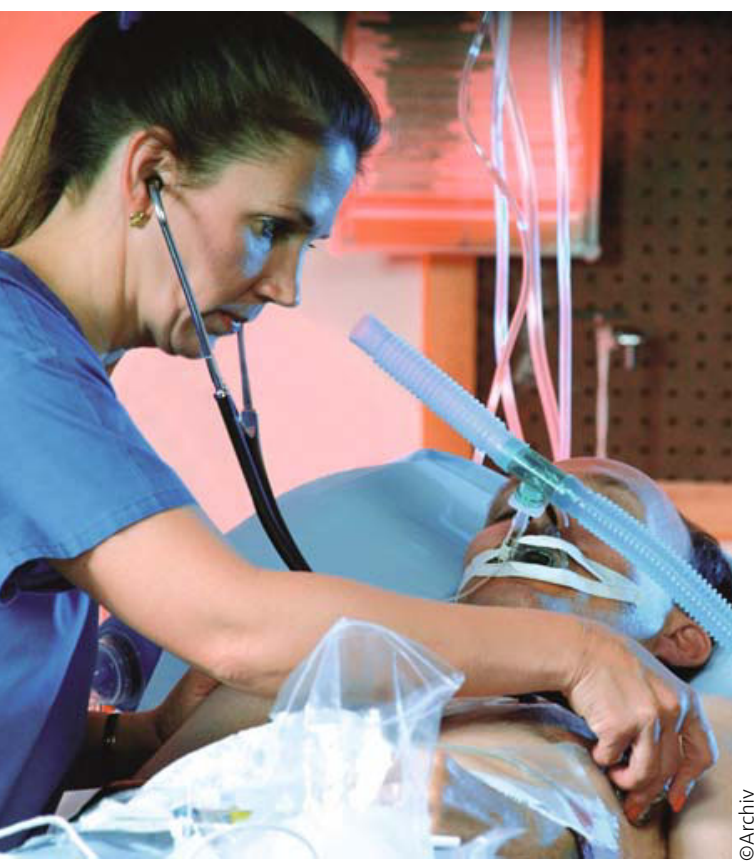

In dieser Situation sind normale Blutdruckwerte eher von Nachteil.

\section{Tabelle 1}

1-Jahres-Mortalität in Abhängigkeit vom systolischen Aufnahmeblutdruck

$\begin{array}{lllll}\text { Syst. BD }(\mathrm{mmHg}) & <128 & 128-144 & 145-162 & >162 \\ \text { Mortalität } & +40,3 \% & \text { Referenz } & -15,2 \% & -21,7 \%\end{array}$

Es besteht eine inverse Korrelation zwischen dem systolischen Blutdruck bei der Aufnahme und der Mortalität nach einem Jahr (s. Tab. 1) und nach drei Jahren. Mögliche Einflussfaktoren wie Alter, Geschlecht, Rauchgewohnheiten, diastolischer Blutdruck, Einnahme von Antihypertensiva und $\mathrm{Ni}$ troglyzerin bei der Aufnahme, aber auch die Behandlung mit Antihypertensiva, Statinen bzw. anderen Lipidsenkern, Thrombozytenaggregations- hemmern und Antikoagulanzien bei der Entlassung wurden berücksichtigt. Die inverse Korrelation gilt auch für Patienten mit der Entlassungsdiagnose koronare Herzkrankheit und für Diabetiker. Sie bleibt auch nach dem Ausschluss aller Patienten mit Herzinsuffizienz und solchen, die während des Klinikaufenthaltes verstorben sind, die also evtl. mit schockbedingter Hypotonie eingeliefert wurden, erhalten.

\section{Kommenta}

Die Autoren haben in zahlreichen Zusatzanalysen versucht, Patienten mit niedrigem Blutdruck infolge lebensbedrohlicher Vor- und Begleiterkrankungen auszuschließen. Trotzdem kann man einwenden, dass der niedrige Blutdruck bei der Aufnahme per se ein dubioses Omen ist und die erhöhte Mortalität dieser Patienten verursacht. Doch dann bleibt ungeklärt, warum die signifikante, inverse Korrelation auch im Bereich normaler und erhöhter Blutdruckwerte, also in den Quartilen 2 bis 4 bestehen bleibt. Bemerkenswert ist auch, dass Patienten mit vorbestehender Behandlung mit Antihypertensiva eine schlechtere, solche mit
Statinen und Antithrombotika eine bessere Prognose haben. Die Autoren betonen, dass ihre Ergebnisse die prognostische Bedeutung des Blutdrucks bei stationärer Aufnahme eindeutig belegen. Dies solle aber vorerst nicht dazu führen, auf die Behandlung erhöhter Blutdruckwerte bei Patienten mit akuten Brustschmerzen zu verzichten.

H. HolzGreve =

\footnotetext{
- U. Stenestrand et al.

Association between admission supine systolic blood pressure and 1-year mortality in patients admitted to the intensive care unit for acute chest pain. JAMA 303 (2010) 1167-1172
}

\section{Promotion / Habilitation. \\ Finden der passenden Professur. www.promotion-d.de Tel.: 0681 / 7097 689, Fax 0681 / 7097691 \\ West Promotionshilfe $\mathrm{GmbH}$}

